\title{
Clinical Practice Guidelines-Is "Regulation" the Answer?
}

Eric Wall, $M D, M P H$

Clinical policies, commonly known as clinical practice guidelines (CPGs), have been around for a very long time. In the past two decades, such guidelines have proliferated both in number and in purpose. Numerous evidence-based guidelines have been developed by international organizations (Cochrane, the National Institute for Health and Care Excellence [NICE], etc.), state and federal bodies (the Agency for Healthcare Research and Quality [AHRQ], U.S. Preventive Services Task Force [USPSTF], individual state health policy departments, etc.), medical specialty organizations, and of course, a wide array of for-profit and not-for-profit quality-focused entities (National Quality Forum, National Committee for Quality Assurance among others). Motivations for guideline development are equally diverse, originating in so-called evidencebased guidance to improve care and extending to reductions in undesirable variations in care, improvements in cost-effective care, and most notably in recent years, enhancing quality of care.

In their wide-ranging discussion of the pitfalls, limitations, and inherent problems with many CPG and their development process, Shaughnessy and colleagues $^{1}$ call for more standardization in the ways CPG are developed, evaluated, and disseminated, suggesting that regulation in some way might reduce inherent self-interest, bias, and clinician confusion. I have spent a fair number of years studying and teaching about clinical policy devel-

From the Department of Family Medicine, University of Washington, Seattle.

Funding: none.

Conflict of interest: none declared.

Corresponding author: Eric Wall, MD, MPH, Department of Family Medicine, University of Washington, 4225 Roosevelt Way NE, Seattle, WA 98195 (E-mail: ewall@uwpn.org).

\section{See Related Commentary on Page 644.}

opment. I have participated in multi-disciplinary practice guideline development groups (sometimes as the only representative from the primary care community). In my administrative roles, I have overseen quality measures assessment. As a practicing family physician, I too have been evaluated according to adherence with a variety of guidelines or performance measures. After all this, I cannot help but wonder whether methodologic transparency and guideline process standardization that may produce "better" guidelines are really at the heart of the matter.

Documented nonadherence to a wide variety of guidelines is widespread. ${ }^{2}$ If improved care outcomes are the goal, then CPGs may be one vehicle to assist in their achievement- if in fact they are used. For guidelines to be useful, they must be relevant to the care setting, clear, easy to access and apply, and auditable for feedback and reporting. That outcomes of care might vary or even be prioritized differently among different patients with very different characteristics has somehow been lost in many evidence-based guidelines. ${ }^{3}$ Randomized controlled trials, which provide highest quality evidence support, are conducted in small and often unrepresentative populations. To make recommendations that apply to most patients who physicians encounter, guideline authors frequently have to extrapolate findings to groups that were not adequately represented in the trials. CPGs exist for a relatively small number of discrete but important conditions found in primary care settings. Even then, few if any CPGs address the typical family medicine patient with multiple health and other issues. Taking this further, research evidence alone is insufficient to support patient decision making. Incorporating patient preferences into clinical decision making is challenged on many fronts. ${ }^{4}$ Highquality evidence informs but does not prescribe patient decisions. Even strong, unambiguous evidence-based recommendations that exclude con- 
textual factors of patient values and goals will be unlikely to achieve desired outcomes.

For CPGs to be useful to physicians, they should be unequivocal in their recommendation and strongly supported by current, high-quality evidence. Given the formal methods used to analyze evidence and estimate outcomes, it is somewhat alarming to still encounter variations in practice recommendations as disparate as the variations in care they are attempting to address. Furthermore, the lack of real guidance as to prioritization of services supported by multiple CPGs in a timelimited patient encounter may also affect adherence. Cost and comparative effectiveness are almost never included in most CPGs even though both are very important to patients.

I am not discounting the enormous importance of evidence-based practice guidelines. I know that we family physicians can all do a better job of critical appraisal and in applying evidence-based care more consistently than we are currently doing. I am less certain that regulation of the guideline development process addresses all the critical barriers to guideline application. Although the characteristics of the guideline itself (relevance, strength of evidence support, clarity, ease of implementation, etc.) certainly are involved, following CPGs involves a complex relationship of individual physician knowledge, attitude, beliefs, and values ${ }^{5}$ along with dissemination/communication strategies, audit/feedback mechanisms, opinion/peer leaders, and a variety of incentives/disincentives, not to even mention, incorporating patient preference. $^{6}$

As if this was not enough, the relationship between CPGs and assessment of clinical performance and quality should be mentioned. The objective of the former (guidelines) is in fact to guide better patient care. Although rewarding quality or clinical performance goals ("pay for performance") clearly incentivizes physician behavior and guideline adherence, the two are not inextricably intertwined. Evidence-based CPGs frame many but not all clinical performance measures. Practice guidelines introduce a level of clinical flexibility (incorporation of patient preferences for example) which is not recognized in measuring clinical performance (was a medication or test given or not and why). ${ }^{7}$
The institutionalization of quality measures by the Centers for Medicare and Medicaid Services and other public and private payers is far more likely to affect performance measures in primary care through a combination of financial incentives and disincentives with guideline transparency, consistency, quality, a secondary concern. Although this has not yielded conclusive evidence of significant improvements in population or individual health, it certainly has moved practice behaviors.

I agree with Shaughnessy and colleagues ${ }^{1}$ that improvements in standardizing or harmonizing CPGs with attention to guideline development, implementation, and evaluation should help reduce physician and patient confusion, improve their trustworthiness, and may even lead to modest improvements in guideline adherence. At the same time we should never forget that caring for our patients is hard work. To that end, family physicians need to be at the table individually and collectively to influence the quality of the practice guidelines that shape the care we provide and to inform guideline developers that engaging patients in shared decision making is the best way to use evidence-based guidelines.

\section{References}

1. Shaughnessy AF, Cosgrove L, Lexchin JR. The need to systematically evaluate clinical practice guidelines. J Am Board Fam Med 2016;29:644-8.

2. Timmermans S, Mauck A. The promises and pitfalls of evidence-based medicine. Health Aff 2005; 24:18-28.

3. Eddy DM, Adler J, Patterson B, Lucas D, Smith KA, Morris M. Individualize guidelines: The potential for increasing quality and decreasing costs. Ann Intern Med 2011;154:627-34.

4. Montori VM, Brito JP, Murad MH. The optimal practice of evidence-based medicine: incorporating patient preferences in practice guidelines. JAMA 2013;310:2503-4.

5. Baiardini I, Braido F, Bonini M, Compalati E, Canonica GW. Why do doctors and patients not follow guidelines? Curr Opin Allergy Clin Immunol 2009;9: 228-33.

6. Institute of medicine (US) committee on standards for developing trustworthy clinical practice guidelines. In: Graham R, Mancher M, Miller Wolman D, et al., eds. Clinical practice guidelines we can trust. Washington, DC: National Academies Press, 2011.

7. Garber A. Evidence-based Guidelines as a Foundation for Performance Incentives. Health Aff 2005;24: 174-9. 